\title{
Descriptive Study on Rehabilitation Treatment and Evaluation Methods for Improving Upper Limb Function in Stroke Patients
}

\section{Youn-Bum Sung ${ }^{1}$, Dae-Hwan Lee ${ }^{1}$, Jung-Ho Lee ${ }^{2}$}

\section{ABSTRACT}

Stroke is a disease in the central nervous system in which sudden ischemia or haemorrhage in blood vessels disrupts the smooth blood supply to brain tissues, resulting in a partial loss of brain function and consequent functional disorders.

Aim and Scope: To improve upper limb function, current stroke interventions employ various treatment methods targeted at the nervous system. Interventions and studies are underway regarding continuous upper limb training programs, such as constraintinduced movement therapy, mirror therapy, imagery training, and robot therapy.

Method: In recent years, treatments using virtual reality systems have also been applied. And various evaluation methods were developed for stroke patients. However, despite the various treatment and evaluation methods introduced thus far, most therapists still insist on applying the methods that they have mainly been using. If the therapist can accurately recognize each treatment/evaluation methods and apply the appropriate treatment method to various situations, a more qualitative treatment can be applied.

Conclusion: This study intends to introduce the existing treatment and evaluation methods of improving stroke patients' upper limb function and find ways for therapists to make better use of them through a proper understanding of each method's characteristics.

Key Words: Stroke, Upper limb rehabilitation, Treatment methods, Evaluation methods

\section{INTRODUCTION}

Stroke is a disease in the central nervous system in which sudden ischemia or haemorrhage in blood vessels inhibit the smooth blood supply to brain tissues, thereby causing a partial loss of brain function and consequent functional disorders. ${ }^{1}$ Stroke patients generally experience neurological disorders, such as motor, sensory, cognitive, language, and emotional disorders. ${ }^{2}$ Particularly, patients with stiffening, muscular weakness, excessive tension, and imbalances in their upper limbs can lose the ability to control their upper limb movement and thus perform only limited social activities and activities of daily living(ADLs). ${ }^{3,4}$ These disorders cause stroke patients to experience lowered self-efficacy, psychological and emotional pains(such as depression) and lowered quality of life. ${ }^{5,6}$ At least $70 \%$ of stroke patients develop upper limb paralysis, and at least $60 \%$ show declines in dexterity. ${ }^{6}$ Stroke patients most commonly recovered from the disease three to six months after its onset. Rehabilitative treatments during this period can facilitate their functional restoration by aiding in neurological recovery. ${ }^{7}$ Within six months after stroke onset, patients can hardly exercise on their own and thus require the assistance of therapists.

Approximately $85 \%$ of stroke patients exhibit cognitive, motor, sensory, and balance disorders, and over $69 \%$ of them develop dysfunctions in their affected upper limbs[8]. Notably, according to previous research, only about $20 \%$ of patients who showed severe motor disorders in their affected upper limbs in the early stages of stroke partially recover their upper limb function. Moreover, around $25 \%$ of stroke patients complain of difficulties in the proper use of their upper limbs even five years after stroke onset, signifying small chances of recovering upper limb function after a stroke.,10 Upper limb disorders occurring after a stroke become a primary cause of the inhibition of movements such as eating,

\section{Corresponding Author:}

Dae-Hwan Lee, Department of Physical Therapy, Daegu Univ., Jillyang-eup, Gyeongsan-si, Gyeongsangbuk-do, Republic of Korea. Email: dhlee8510@naver.com

ISSN: 2231-2196 (Print)

Received: 15.07 .2020
ISSN: $0975-5241$ (Online)

Revised: 18.08 .2020
Accepted: 20.09 .2020
Published: 06.10 .2020 
walking, handwriting, balancing, manipulation, delicate hand movements, personal hygiene management. ${ }^{11}$ Stroke patients' recovery of their upper limb function is important for them to maintain the most basic human life. This function may be as crucial for performing ADLs as the lower limb's gait function. ${ }^{12}$

The reason for focusing on upper limb rehabilitation out of the various issues that stroke patients can experience is that impairment of the middle cerebral artery, which is in charge of upper limb function, accounts for $75 \%$ of all stroke cases. ${ }^{13}$ A stroke patient's weakness in the upper limb increases his/her reliance on others by lowering their independence in performing ADLs. Particularly, a patient whose weakened upper limb and major hand are on the same side needs assistance in most ADLs, including eating, face washing, toilet use, dressing, and grooming. Therefore, not only stroke patients but also their caregivers, who should stay close to them, can experience declines in their quality of life. Stroke patients' upper limb rehabilitation should aim to reduce the spread of brain damage during the acute treatment period and mitigate the disorders resulting from aftereffects through the problem-solving process after the acute stage. ${ }^{13,14}$

At present, stroke interventions employ various methods to improve upper limb function, including Bobath therapy, proprioceptive neuromuscular facilitation (PNF), and taskoriented training. Also, interventions and studies are underway for upper limb training programs, such as constraintinduced movement therapy (CIMT), and mirror therapy. In recent years, treatments using the robot and virtual reality systems have also been adopted. ${ }^{15}$ Also, the manual function test (MFT), Brunnstrom recovery stage(BRS), FuglMeyer assessment for the upper limb(FMA-U/L), Korean modified Barthel index(K-MBI), motor activity $\log (\mathrm{MAL})$, box and block test(BBT),two-point discrimination test, tactile detection threshold test, thermal sensory threshold test, vibration sensory threshold test, upper limb muscle activity test, and electroencephalography (EEG) are mainly used as upper limb diagnosis and evaluation methods for stroke patients.

However, despite the wide range of treatment and evaluation methods introduced to date, many therapists cling to the methods that they have mainly been using. If the therapist can accurately recognize each treatment or evaluation method and apply the appropriate treatment method to various situations, a more qualitative treatment can be applied. Therefore, this study intends to introduce the existing treatment and evaluation methods of improving stroke patients' upper limb function and find ways for therapists to make better use of them through a proper understanding of each method's characteristics.

\section{METHODS}

This study found and compiled papers on treatment or evaluation method of stroke patients posted on the Pub-Med since 2000.

\section{Treatment methods of improving stroke pa- tients' upper limb function}

Bobath Therapy: The general purpose of Bobath therapy is to establish treatment programs that enable an individual to withstand the pressure from repeated active exercises, gravity, weight, or hands; achieve a balance between muscle groups, and reduce the effects of abnormal tension in exercise patterns and autonomous responses. ${ }^{16}$

The principle of Bobath therapy is to inhibit abnormal types of sensory stimulations in the sensorimotor system's abnormal closed loops. This therapy aroused normal sensory stimulations as much as possible, and facilitate the maximum level of normal sensory inputs, thereby preventing the vicious cycle of abnormally closed loops. To activate this inhibition, reflex inhibitory postures were developed. ${ }^{13}$ Bobath therapy addresses the particular problem wherein abnormal and widespread reflex patterns of exercises and postures occur from the brain stem, cerebellum, midbrain, basal ganglia, and cortex through their disassociation from inhibition. Therefore, this therapy is based on an approach to normal development, motion compensation, and habitual exercise patterns that can cause muscular imbalances or contractions. ${ }^{16}$

The underlying concepts of Bobath therapy are autonomous postural adaptation, sensory structures for inducing postural responses, muscle tone, fixation aimed at compensating for low muscle tone, development of motility, cognition of kinetic potentials, development of active muscles, and supply of muscle control. ${ }^{12}$ Bobath therapy uses functional activities and teaches how to process muscle tensions. It values the quality of movements in every activity, encourages patients to perform on their own as much as possible, and emphasizes early treatment. It even teaches patients with cerebrovascular diseases to maintain proper postures in bed to enable them to recognize and use their affected sides. It also trains patients in skills to move quickly in bed as early as possible to help them avoid movement patterns that strengthen abnormal reflexes. ${ }^{16}$

Therapists should control patients' movements to generate normal patterns and teach them to sense such movements. Patients are also instructed to reduce the range of movements controlled by therapists and replace therapists' control with their own. Key points of control are used to promote normal movements. Initially, key points of control are mostly in the body's proximal parts, such as the trunk and the pelvis. As their conditions improve, patients learn to use the distal parts of their bodies as the core of control. Therapists should con- 
trol the intensity of therapy to promote patients' control of their movements. ${ }^{17}$

Proprioceptive Neuromuscular Facilitation (PNF): PNF treatment aims to increase the potential memory of movement patterns that are as normal as possible in the central nervous system. This is achieved by providing a sufficient level of early aids (proprioceptive, foreign, and terminalreceptive signals) to arouse patients' needs and responses. Self-reliance means patients' deferred tasks for future use and already learned tasks. ${ }^{16}$

PNF involves spiral and diagonal mass movement patterns. ${ }^{18}$ These patterns are a combination of exercises in the sagittal, coronal, and cross-sectional planes. Specifically, PNF combines flexion and extension in the sagittal plane, pronation and supination in the coronal plane, and rotation in the cross-sectional plane. This combination of functional movements increases muscle activities and spreads them toward the proximal and distal areas. ${ }^{19}$

These characteristics can explain Beevor's principle: 1) PNF uses group movement patterns that become the basis of all techniques. 2) Mass muscular movement patterns are the original trait of normal movements.3) The brain is not aware of any individual muscle action except the movements that occur. Mass movement patterns can be classified as the upper limb, lower limb, trunk, cephalic, and temporomandibular patterns. These patterns vary by the joint(axis) from which movements mainly occur. When each pattern is clinically used, the combination of rhythmic inception, rhythmic stability, repeated contraction, the timing of emphasis, reversed motions by the antagonist, slow reversal, the reversal of the antagonist and the agonist, contraction-relaxation, maintenance-relaxation, and isotonic contraction can result in numerous variations of a single pattern. ${ }^{20}$

The locations and positions of patients and therapists, verbal orders for auditory stimulations, use of patients' vision, extension and compression, timing, and movement patterns are applied in methods and procedures of applying the facilitation, proper resistance, use of diffusion and reinforcement, and barehand contact for cognitive and tactile simulations. ${ }^{19}$

Task-Oriented Movement Therapy: In the 1980s, selective task-oriented training was developed as a treatment technique for stroke patients based on motor learning theory. This training assumes that the provision of functional tasks to patients and their active problem solving are more effective for learning than the repeated training of a single movement. ${ }^{21}$ Its task-oriented approach to motor recovery characterizes selective task-oriented training. It is also based on dynamic system theory, which explains the performance of the tasks and motor recovery that occur in the dynamic interactions between individuals, environments, and tasks that they perform. ${ }^{22}$
Stroke patients' selective task-oriented training is goal-oriented; it helps them achieve their goals by performing movements within their daily environments through the provision of functional tasks. It is also an effective treatment technique through which stroke patients can expect functional recoveries. ${ }^{23}$ Patients' full engagement in an activity or task from the beginning to the end can elicit stronger, more effective, and coordinated motor responses than partial engagement. ${ }^{24}$ Previous research on task-oriented training shows that tasks and intervention strategies that provide meaningful activities related to daily living are more helpful than repeated or passive exercises for learning skills and improving functions. ${ }^{25}$ A study on selective task-oriented training reports that task training focused on patients' preferred tasks or goals facilitates changes in ADLs and subsequently produced more effective outcomes from rehabilitation treatments. ${ }^{26}$

Constraint-Induced Movement Therapy (CIMT): CIMT is a behavioural neuroscience-based treatment method used to enhance a stroke patient's motor performance on the affected side in the field of neurorehabilitation. ${ }^{27}$ Stroke patients mostly experience difficulties in using their affected upper limbs and thus learn patterns using their unaffected sides to compensate for such insufficient motions. Continued compensatory learning hinders their intrinsic recovery. ${ }^{28}$ Taub et al. (1980) explained this phenomenon using the term "learned nonuse". In their study, they blocked the vision of monkeys in one side and had them wear jackets designed to constrain their emotions and suppress their compensatory patterns in the unaffected healthy upper limb; as a result, the monkeys used the affected side. Based on this principle, CIMT was introduced as a treatment that enables the learning of functional movement patterns by limiting the use of the unaffected side and inducing intensive movements on the affected side to control learned nonuse. In recent years, it has further evolved into the concept of task-oriented movement therapy. ${ }^{27}$

CIMT is offered to stroke patients to enhance hemiplegic patients' upper limb function by overcoming the learned nonuse that occurs in the acute stage after stroke onset and inducing changes in the cerebral cortex.${ }^{29} \mathrm{CIMT}$, which constrains the unaffected side, increases the use of the affected side through continuous and repeated practices of functional arm movements. Recent studies explain this therapy concerning changes in movement patterns, which are explained through kinetic analyses, and local terminal sprouting or functional synaptic reorganization achieved by stimulating the undamaged cortical circuit and the sensory cortex adjacent to the damaged area. ${ }^{30,31}$

Mirror therapy: Contradicting the basic concept that sensorimotor training is effective for motor skills, mirror therapy is based on the facilitation of the mirror neuron system and induces improvements in the affected side's movements by 
providing visual information. ${ }^{32}$ This therapy was introduced by Ramachandran (1994) for phantom pain reduction in amputees. Its effects on reducing pain and stiffening have been reported. ${ }^{33}$ Mirror therapy was also adopted by Altschuler, Wisdom, and Stone (1999) as a treatment to restore stroke patients' upper limb function. ${ }^{34}$ Moreover, a systematic review by Pollock et al. (2014) defined mirror therapy in the rehabilitation of stroke patients as a visual stimuli-based technique that creates an illusion of movements on the affected side through the mirror reflection of the actual upper and lower limb movements on the unaffected side for the performance of tasks. ${ }^{35}$ Mirror therapy positively affects stroke patients' upper limb function and ability to perform ADLs. ${ }^{36}$

The neurological mechanism of mirror therapy is highly associated with the superior temporal sulcus, and the activation of the primary motor area is exhibited through magnetic resonance imaging. ${ }^{37}$ Also, Thirumala et al. (2002) validated the effects of mirror therapy via mirror neurons by confirming increased stimulations in the primary motor cortex and both inferior parietal lobules during observation of upper limb and hand movements via the mirror. ${ }^{38}$ According to a review of previous research on mirror therapy, recent practices of mirror therapy have shifted from the original movementbased therapy, which comprised forearm, wrist, and knuckle motions, toward task-oriented therapy. ${ }^{39}$

Imagery training: Imagery training teaches patients to learn motor skills and improve their abilities to perform exercises by imagining body movements and repeatedly practising their tasks without performing actual movements. ${ }^{40}$ According to the results of the brain imaging study of Malouin et al. (2004), this imagery training activates a certain area of the cerebral cortex in a similar pattern for performing actual activities, as well as the corticospinal tract. They noted that this implies cerebral plasticity. ${ }^{41}$ Also, during imagery training, the target muscle shows the occurrence of electromyographic (EMG) signals in a similar pattern to actual exercises; furthermore, blood flow and potential increases are observed in the brain area related to actual exercises. ${ }^{42}$ Pollock et al. (2014) stated in their systematic review that the type of imagery training that is most commonly used to rehabilitate stroke patients involves performing physical task-based activities using the affected area after undergoing mental imagery training for certain tasks. ${ }^{35}$

Park and Heo (2014) argued that this type of imagery training is more effective for the recovery of upper limb function when it is accompanied by general treatment than when it is conducted alone. ${ }^{43}$ Decety and Grkzes (1999) stated that the inclusion of exercises tasks focused on meaningful and goal-oriented movements is important for patients who have a clear goal for their exercise tasks during imagery training. ${ }^{44}$

Robot therapy: Over the recent 20 years, robot-assisted re- habilitation has developed into training targeted at improving stroke patients' upper limb and ADL functions. ${ }^{45,46}$ Since the 1990s, various robotic rehabilitation devices have been developed and adopted in the US and Europe. As a result, many studies have reported the effects of these devices. ${ }^{47} \mathrm{Re}-$ habilitation robots help increase the frequency and duration of rehabilitation training. Moreover, independent training of the body's distal ends, such as the hands and wrists, produces better training effects than does conventional training. ${ }^{48}$

Neurological rehabilitation through the enhancement of brain plasticity and direct rehabilitation of joints and muscles for the relaxation of contracture and stiffening should be conducted simultaneously to rehabilitate the upper limbs of hemiplegic stroke patients. Rehabilitation robots reduce therapists' intensity of labour and treatment time, provide precise and consistent treatments, and improve the efficiency and quality of rehabilitative treatments. Specifically, robot training using visual perceptions has certain advantages. It enables patients to spontaneously and actively participate and motivates them to improve their training results through precise and various types of sensory feedback; robot training also trains patients in intensive practices through functional improvements using important tasks. ${ }^{49,50}$ Furthermore, robotbased rehabilitation offers patients various therapeutic training programs through protective equipment and simple operations, thereby challenging and encouraging patients and helping them concentrate on specific tasks. ${ }^{51}$

\section{Evaluation methods for improving stroke pa- tients' upper limb function}

Manual Function Test (MFT): The MFT is frequently used in clinical practice as a testing tool for objectively assessing the overall functional conditions of upper limb motor skills. The MFT for stroke was developed to measure stroke patients' upper limb function and movement ability. It comprises three items: upper limb exercise, grasping, and finger manipulation. Each successful performance is graded 1 point; each failure, 0 . These scores are added to produce a total score based on a 32-point scale and then converted into a final score out of 100 points. The MFT is divided into eight items in three domains, namely, upper limb motor skills (four items), grasp (two items), and finger manipulation (two items), which indicate the recovery conditions of upper limb motor skills. This evaluation tool's test-retest reliability and inter-rater reliability are $\mathrm{r}=0.95$ and a Cronbach's alpha coefficient of $0.95 .^{52}$

Brunnstrom Recovery Stage (BRS): In 1970, Brunnstrom divided the recovery of the proximal upper limb and the hand into six stages. Spontaneous motor recovery generally follows the ontogenic process from the proximal to the distal part. In other words, shoulder movements appear earlier than hand movements, and reflexive motions are controlled and appear earlier than voluntary motions. Although general 
stroke patients recover according to this process, some patients can stop their recoveries at certain stages under the influence of their sensory, perceptive, cognitive, motivational, and emotional conditions or other secondary medical issues. These stages do not indicate the reliability that patients will certainly recover their voluntary movements, and the recovery stages of the upper limb and the hand do not always coincide. $^{53}$

Fugl-Meyer Assessment for the Upper Limb (FMA-U/L): The FMA-U/L is a motor function evaluation tool that follows Brunnstrom's six recovery stages and specifically assesses the body's structure, functions, and activity levels. ${ }^{54}$ It is the most widely used evaluation tool for the upper limb, and it measures proximal and distal upper limb movements by combining the items of shoulder/elbow movement and wrist/hand movement. ${ }^{55}$

This test consists of 33 upper limb-related test items with a total score of 66 points. Its intra-rater reliability is high $(0.95$ to 1.0$).{ }^{56}$ The inter-rater reliability of raters and therapists is also high. The reliabilities for the motor and sensory scores are $r=0.98$ and $r=0.93$, respectively. ${ }^{57}$ To evaluate participants, raters order them to perform certain movements and observe their responses. Of the total of 50 items, only 33 items related to the upper limb are used, which comprise 18 items for the shoulder/elbow, five items for the wrist, seven items for the hand/finger, and three items for the upper limb's coordination. Each participant is given 2 points for a complete performance, 1 point for partial performance and 0 points for failure and all points are added. ${ }^{55}$

Korean Modified Barthel Index (K-MBI): The K-MBI is used to evaluate ADLs. The MBI that Granger et al. (1979) produced by modifying and improving the Barthel index is sensitive to functional changes. The K-MBI comprises 10 items: seven items for daily personal activities (such as personal hygiene, bathing, eating, toilet use, going up and downstairs, dressing, bowel and bladder control, ambulation, wheelchair use, and chair and bed transfer) and three items for operating skills. ${ }^{59}$ Raters ask participants to perform each of these items and then observe their responses. Each movement is given a score out of 100 points based on five stages: 0 to 20 points for complete dependence, 21 to 61 points for heavy dependence, 62 to 90 points for moderate dependence, 91 to 99 points for mild dependence, and 100 points for complete independence. The intra-rater reliability is $\mathrm{r}=0.89$, and the inter-rater reliability is $\mathrm{r}=0.95 .^{58}$

Motor Activity Log (MAL): $\quad$ The MAL is a structured interview-based evaluation tool that aims to understand how stroke patients use their affected upper limbs in daily living both qualitatively and quantitatively. ${ }^{60}$ It has 30 questions that are divided into qualitative and quantitative measures, and the level of each performance is scored on a scale of 0 to 5 . The full score of istherefore 150 points. The quantitative measures evaluate the level of usage of the paralyzed upper limb in daily life, whereas the qualitative measures assess the improvement level of the quality of movement during exercise. The MAL is a highly reliable test tool with an interitem consistency of $r=0.88$ to 0.95 , inter-rater reliability of $\mathrm{r}=0.90$, and test-retest reliability at $\mathrm{r}=0.94$.

Box and Block Test (BBT): As a tool for evaluating the quickness of the upper limb, the BBT comprises a wood cube measuring $2.54 \mathrm{~cm}$ in length and a $53.7 \times 8.5 \times 27.4 \mathrm{~cm} \mathrm{rec}-$ tangular box divided into two square compartments of equal dimension using a middle partition. Its intra-rater reliabilities are $\mathrm{r}=0.99$ for the left hand and $\mathrm{r}=0.94$ for the right hand. The inter-rater reliabilities are $\mathrm{r}=0.99$ for the left hand and $\mathrm{r}=1.00$ for the right hand. ${ }^{61}$ Participants conduct the main test for one minute after a 15 -second rehearsal. The wood cubes that each participant picks up and moves to the opposite side with his/her affected hand are counted.

Two-Point Discrimination Test: This test measures the ability to perceive two stimulations given simultaneously. It mainly uses the standardized tool Dellon2-Point Disk-Criminator. The two-point discrimination test mostly targets the index finger or fingertip. It is conducted while participants are sitting in a quiet space with little distraction and minimal noises from the rater or the testing tools. During the test, the patients' eyes are covered, and they are instructed to assume a comfortable posture while exposing the tested body region. The rater asks the patients to tell whether they are in contact with one or two disk points according to their perception. The rater runs the test until a patient cannot perceive two points through modifying the testing range by 1 mmeach time for the hand. The smallest distance between two points that the patient can distinguish is defined as the two-point threshold.

Tactile Detection Threshold Test: This test generally uses the Semmes-Weinstein monofilament (SWM), which is a standardized tool in which patients identify light tactile stimulations and their locations. The SWM is a sensory set of five monofilaments with weights of $0.07 \mathrm{~g}(2.831 \mathrm{~b}), 0.4 \mathrm{~g}(3.61 \mathrm{lb})$, $2.0 \mathrm{~g}(4.31 \mathrm{lb}), 4.0 \mathrm{~g}(4.561 \mathrm{~b})$, and $300 \mathrm{~g}(6.65 \mathrm{lb})$; these are attached to a rod that is long enough to be handheld. The index finger and the fingertip are mostly measured. The test starts from the heaviest monofilament (6.65lb) and stops when the patient can no longer perceive contact. The weight of the last monofilament sensed by the patient is recorded.

Thermal Sensory Threshold Test: In this test, a temperature sensor is attached to the patient's affected upper limb. The standard temperature is $32^{\circ} \mathrm{C}$, with an increase or decrease of $1{ }^{\circ} \mathrm{C}$ per second. Each patient is instructed to press a mouse button when feeling a cool or warm sensation for the first time. A computer measures the temperatures during these button presses and a change in the current direction restores the sensor's temperature to the standard level. In the 
temperature range of $0^{\circ} \mathrm{C}$ to $50^{\circ} \mathrm{C}$, the thresholds of cool and warm sensations are recorded. If a patient does not feel a cool sensation at $0^{\circ} \mathrm{C}$, then the threshold is measured at $0^{\circ} \mathrm{C}$. Similarly, if the patient does not feel a warm sensation at $50^{\circ} \mathrm{C}$, then the threshold is set at $50^{\circ} \mathrm{C}$. For the measurement, the testing environment should be created in consideration of patients' psychological and physiological characteristics. The temperature should be in the pleasant range of $18^{\circ} \mathrm{Cto}$ $2{ }^{\circ} \mathrm{C}$. Space should be free from distractions so that patients can undergo the test without disturbance in a comfortable position while holding the feedback unit in one hand.

Vibration Sensory Threshold Test: To measure quantitative sensory vibration thresholds, this test increases the intensity of stimulations using a handheld vibrator until a vibratory sensation is felt in the index finger and the forearm on the affected side. The rater gradually increases the intensity of a $100 \mathrm{~Hz}$ vibration at a speed of $1 \mu \mathrm{m} / \mathrm{s}$ and instructs the patient to press a button the moment they first sense a vibration. An optimal testing environment should be created by considering patients' psychological and physiological characteristics. For example, they should be allowed to keep their arms in a comfortable position during the test. They should sit $1.5 \mathrm{~m}$ away from the screen and be prevented from looking at it. They should also be fully educated and perform a rehearsal before the test. A mean value of three measurements should be used for data analysis.

Upper Limb Muscle Activity Test: To measure surface EMG(sEMG) signals for EMG analysis using root-meansquare (RMS) values. This test employs an EMG instrument that can measure various bio-signals from muscle transposition with a high input impedance and a common-mode rejection ratio of $100 \mathrm{~dB}$ or above. When measuring bio-signals, they are digitized at a frequency of $250 \mathrm{~Hz}$ and quantized at 24 bits. The subject's body areas on both the affected and unaffected sides can be measured, such as the biceps brachii, triceps brachii, wrist extensor, and wrist flexor. $\mathrm{An} \mathrm{Ag}-\mathrm{AgCl}$ electrode is used for sEMG, and surface electrodes are positioned with an interelectrode distance of $20 \mathrm{~mm}$. TheEMG signals measured in the electrodes are immediately amplified 10,000 times after going through an amplifier and then transmitted to a terminal through digitization and quantization. Regarding the data measured in the terminal, the automatic indication of raw data is confirmed using the software BioScan, and a filtering process is then performed by setting a $60 \mathrm{~Hz}$ notch filter and a band-pass filter in the range of 0.5 to $250 \mathrm{~Hz}$. For each movement, each muscle's RMS values are calculated for data and statistical analyses.

Electroencephalography (EEG): The brainwaves of subjects are stored in a computer using a sampling frequency of $256 \mathrm{~Hz}$, a $0.5-50 \mathrm{hHzpass}$ filter, and 12-bit AD conversion. Brainwave data are collected using electrodes attached to the head. These electrodes are attached to the scalp positions
Fp1, Fp2, F7, F3, FZ, F4, F8, T3, C3, CZ, C4, T4, T5, P3, $\mathrm{PZ}, \mathrm{P} 4, \mathrm{~T} 6, \mathrm{O} 1$, and $\mathrm{O} 2$ in accordance with the international 10-20 method of electrode placement. Reference electrodes are attached below each ear.

EEG electrodes are disk electrodes in the form of a goldcoated plate. To minimize the skin contact resistance in the attachment of electrodes, any foreign substances in the scalp should be wiped with alcohol swabs prepared in advance. Then, the electrodes are attached using electrode paste. The attached electrodes should be fixed to prevent detachment by covering them with gauze. After the attachment of electrodes, subjects are measured while sitting in a comfortable chair in a quiet, noise-blocked test room. For data processing, signals are collected by measuring spontaneous potentials for three minutes and evoked potentials for 15 seconds and then repeating this set of measurements 30 times. During the collection of brainwave data, five seconds of data measured at the beginning and the end are removed to exclude the possibility of momentary declines in the subject's attention or the inflow of artefacts. The BioScan program is used for the digital conversion and analysis of brainwaves. Brainwave experts analyze signals sent to the computer to output indicators. A brain mapping program that visualizes the relative alpha and beta values of each region's brain waves is employed to visually monitor brainwave changes for each brain region under the activation of brainwaves.

\section{DISCUSSION}

Stroke is one of the 10 leading causes of death and one of the three major diseases in South Korea. The number of deaths from stroke is 23,415 per 100,000 people, accounting for $45.8 \%{ }^{62}$ Stroke is a neurological condition generated by the discontinuance of blood supply due to the rupture of blood vessels or their blockage by blood clots. This disease impairs brain tissues by blocking the supply of oxygen and nutrients. ${ }^{63}$ Its symptoms include motor, sensory, cognitive, emotional, and speech disorders. ${ }^{64}$ About $16 \%$ to $30 \%$ of stroke patients show loss of cognitive functions, such as orientation, attention, memory, language skills, and visuospatial ability, within one year after stroke onset. These disorders cause difficulties in performing independent ADLs, including bathing, travelling, and bowel and bladder control. ${ }^{65,66}$ Because most ADLs are linked to upper limb function, the recovery of upper limb function has a significant impact on stroke patients. Multiple studies assert that upper limb function is restored in the early stages of stroke. However, Taub et al. (1993) stated that some patients recovered their upper limb function until one year after stroke onset. ${ }^{27}$ They reported that, although quick recoveries are made in the early stages of rehabilitation, active functional recoveries require more time. ${ }^{21}$ Therefore, therapeutic approaches aimed at enhancing upper limb function are important. ${ }^{67}$ 
This study found and compiled papers on treatment or evaluation method of stroke patients posted on the Pub-Med since 2000. The principle of Bobath therapy is to inhibit abnormal types of sensory stimulations in the sensorimotor system's abnormal closed loops. This therapy aroused normal sensory stimulations as much as possible, and facilitate the maximum level of normal sensory inputs, thereby preventing the vicious cycle of abnormally closed loops. PNF involves spiral and diagonal mass movement patterns. These patterns are a combination of exercises in the sagittal, coronal, and cross-sectional planes. Specifically, PNF combines flexion and extension in the sagittal plane, pronation and supination in the coronal plane, and rotation in the cross-sectional plane. This combination of functional movements increases muscle activities and spreads them toward the proximal and distal areas. Task-oriented movement therapy assumes that the provision of functional tasks to patients and their active problem solving are more effective for learning than the repeated training of a single movement. Its task-oriented approach to motor recovery characterizes selective task-oriented training. It is also based on dynamic system theory, which explains the performance of the tasks and motor recovery that occur in the dynamic interactions between individuals, environments, and tasks that they perform. Constraint-Induced Movement Therapy (CIMT) is offered to stroke patients to enhance hemiplegic patients' upper limb function by overcoming the learned nonuse that occurs in the acute stage after stroke onset and inducing changes in the cerebral cortex. CIMT, which constrains the unaffected side, increases the use of the affected side through continuous and repeated practices of functional arm movements. Recent studies explain this therapy concerning changes in movement patterns, which are explained through kinetic analyses, and local terminal sprouting or functional synaptic reorganization achieved by stimulating the undamaged cortical circuit and the sensory cortex adjacent to the damaged area. Lastly contradicting the basic concept that sensorimotor training is effective for motor skills, mirror therapy is based on the facilitation of the mirror neuron system and induces improvements in the affected side's movements by providing visual information. Mirror therapy focuses on the fact that visual stimulations are effective for improving motor skills. ${ }^{67}$

\section{CONCLUSION}

In the present study, various treatment methods were investigated as stroke interventions to improve upper limb function. They included the MFT, BRS, FMA-U/L, K-MBI, MAL, BBT, two-point discrimination test, tactile detection threshold test, thermal sensory threshold test, vibration sensory threshold test, upper limb muscle activity test, and EEG. Also, leading treatment methods were reviewed, including Bobath therapy, PNF, task-oriented training, CIMT, mirror therapy, imagery training, and robot therapy. It is difficult to state that certain treatment/evaluation methods are superior to others. Nevertheless, the flexible applications of the various treatment or evaluation methods presented by this study to suit varied circumstances will induce effective treatments for the recovery of stroke patients' overall physical functions, thereby ultimately enhancing their upper limb function.

\section{ACKNOWLEDGEMENTS}

This work was supported by the National Foundation of Korea (NRF) grant funded by the Korea government (MSIT) (No-2019R1F1A1057731).

\section{Conflict of Interest: Nil}

\section{Source of Funding: Nil}

\section{REFERENCES}

1. Prange GB, Jannink MJ, Groothuis-Oudshoorn CG, Hermens HJ, Ijzerman MJ. A systematic review of the effect of robot-aided therapy on recovery of the hemiparetic arm after stroke. Journal of rehabilitation research and development. 2009;43(2):171-84.

2. Radomski MV, Latham CA, editors. Occupational therapy for physical dysfunction. Lippincott Williams \& Wilkins; 2008.

3. Gracies JM, Marosszeky JE, Renton R, Sandanam J, Gandevia SC, Burke D. Short-term effects of dynamic lycra splints on upper limb in hemiplegic patients. Archives of physical medicine and rehabilitation. 2000 Dec 1;81(12):1547-55.

4. Dijkerman HC, Ietswaart $M$, Johnston $M$, MacWalter RS. Does motor imagery training improve hand function in chronic stroke patients? A pilot study. Clinical rehabilitation. 2004 Aug;18(5):538-49.

5. Robinson-Smith G, Johnston MV, Allen J. Self-care self-efficacy, quality of life, and depression after stroke. Arch. Phy. Med. Reha.APMR). 2000 Apr 1;81(4):460-4.

6. Mee-sook Cheong and Bong-hee Jin. The Effect of Ayurvedic Color Therapy Program on Infant Attention Deficit. Inter. J. Child Warfare Promo. Manag. 2018 Apr;2(1):7-12.

7. Luke C, Dodd KJ, Brock K. Outcomes of the Bobath concept on upper limb recovery following stroke. Clinical rehabilitation. 2004 Dec;18(8):888-98.

8. Geddes JM, Fear J, Tennant A, Pickering A, Hillman M, Chamberlain MA. Prevalence of self-reported stroke in a population in northern England. Journal of Epidemiology \& Community Health. 1996 Apr 1;50(2):140-3.

9. Hayward K, Barker R, Brauer S. Interventions to promote upper limb recovery in stroke survivors with severe paresis: a systematic review. Disability and rehabilitation. 2010 Jan 1;32(24):1973-86.

10. Soo-yeon Jeong, Kwangho Lee. 2nd Graders' N-Back Training Performance. International Journal of Child Warfare Promotion and Management. 2018 Oct;2(2):19-24.

11. Sheng B, Lin M. A longitudinal study of functional magnetic resonance imaging in upper-limb hemiplegia after stroke treated with constraint-induced movement therapy. Brain injury. 2009 Jan 1;23(1):65-70.

12. Markus H, Pereira A, Cloud G. Stroke medicine. Oxford University Press; 2016 Dec 1. 
13. P. Langhome, J. Bernhardt, G. Kwakkel. Post Stroke Rehabilitation. The Lancet. 2011;377(9778):1693-1702.

14. Bird ML, Cannell J, Jovic E, Rathjen A, Lane K, Tyson A, Callisaya $\mathrm{M}$, Smith S. A randomized controlled trial investigating the efficacy of virtual reality in inpatient stroke rehabilitation. Archives of Physical Medicine and Rehabilitation. 2017 Oct 1;98(10):e27.

15. Hang-Jun Choi. The Influences of Mother's Psychological characteristics on Marital Relationship: The Mediating Effect of Preschooler's Effortful Control. International Journal of Child Warfare Promotion and Management(IJCWPM). 2018 Apr;2(1):19-26.

16. Platz T, Eickhof C, Van Kaick S, Engel U, Pinkowski C, Kalok S, Pause M. Impairment-oriented training or Bobath therapy for severe arm paresis after stroke: a single-blind, multicentre randomized controlled trial. Clinical rehabilitation. 2005 Nov;19(7):714-24.

17. Voss DE, Ionta MK, Myers BJ, Knott M. Proprioceptive neuromuscular facilitation: patterns and techniques. Philadelphia, PA: Harper \& Row; 1985.

18. Buck M, Beckers D, Adler S. PNF in Practice. Springer-Verlag Berlin Heidelberg. New York. 1993.

19. S. Adler, D. Beckers, and M. Buck. Patterns of Facilitation. In: PNF in Practice. Springer. Berlin. Heidelberg. 2000:57-62.

20. J. Carr, and R. Shepherd. Stroke rehabilitation: guidelines for exercise and training to optimize motor skill. Butterworth-Hein emann Medical. 2003.

21. Ciro CA, Hershey LA, Garrison D. Enhanced task-oriented training in a person with dementia with Lewy bodies. American Journal of Occupational Therapy. 2013 Sep 1;67(5):556-63.

22. G. Thielman, C. Dean, A. Gentile. Rehabilitation of reaching after stroke: task-related training versus progressive resistive exercise. Archives of Physical Medicine and Rehabilitation. 2014;85(10):1613-18.

23. Pendleton HM, Schultz-Krohn W. Pedretti's Occupational therapy-e-book: Practice skills for physical dysfunction. Elsevier Health Sciences; 2017 Mar 10.

24. Winstein CJ, Rose DK, Tan SM, Lewthwaite R, Chui HC, Azen SP. A randomized controlled comparison of upper-extremity rehabilitation strategies in acute stroke: a pilot study of immediate and long-term outcomes. Archives of physical medicine and rehabilitation. 2004 Apr 1;85(4):620-8.

25. Spooren AI, Janssen-Potten YJ, Kerckhofs E, Seelen HA. The outcome of motor training programmes on arm and hand functioning in patients with cervical spinal cord injury according to different levels of the ICF: a systematic review. Journal of rehabilitation medicine. 2009 Jun 5;41(7):497-505.

26. Taub E, Uswatte G, Pidikiti R. Constraint-induced movement therapy: a new family of techniques with broad application to physical rehabilitation-a clinical review. Journal of rehabilitation research and development. $1999 \mathrm{Jul}$ 1;36(3):237-51.

27. Dromerick AW, Edwards DF, Hahn M. Does the application of constraint-induced movement therapy during acute rehabilitation reduce arm impairment after ischemic stroke?. Stroke. 2000 Dec 1;31(12):2984-8.

28. Fritz SL, Light KE, Clifford SN, Patterson TS, Behrman AL, Davis SB. Descriptive characteristics as potential predictors of outcomes following constraint-induced movement therapy for people after stroke. Physical therapy. 2006 Jun 1;86(6):825-32.

29. Nelles G, Jentzen W, Jueptner M, Müller S, Diener HC. Arm training-induced brain plasticity in stroke studied with serial positron emission tomography. Neuroimage. 2001 Jun 1;13(6):1146-54.

30. Rossini PM, Pauri F. Neuromagnetic integrated methods tracking human brain mechanisms of sensorimotor areas 'plastic reor- ganisation. Brain research reviews. 2000 Sep 1;33(2-3):131-54. 31. H. Thieme, M. Bayn, M. Wurg, C. Zange, M. Pohl, J. Behrens. Mirror therapy for patients with severe arm paresis after stroke-randomized controlled trial. Clinical rehabilitation. 2013;27(4):314-24.

32. Ramachandran VS. Phantom limbs, neglect syndromes, repressed memories, and Freudian psychology. International review of neurobiology. 1994 Jan 1:291-291.

33. Altschuler EL, Wisdom SB, Stone L, Foster C, Galasko D, Llewellyn DM, Ramachandran VS. Rehabilitation of hemiparesis after stroke with a mirror. The Lancet. 1999 Jun 12;353(9169):2035-6.

34. Pollock A, Farmer SE, Brady MC, Langhorne P, Mead GE, Mehrholz J, van Wijck F. Interventions for improving upper limb function after stroke. Cochrane Database of Systematic Reviews. 2014(11).

35. Invernizzi M, Negrini S, Carda S, Lanzotti L, Cisari C, Baricich A. The value of adding mirror therapy for upper limb motor recovery of subacute stroke patients: a randomized controlled trial. Eur J Phys Rehabil Med. 2013 Jun 1;49(3):311-7.

36. Matthys K, Smits M, Van der Geest JN, Van der Lugt A, Seurinck R, Stam HJ, Selles RW. Mirror-induced visual illusion of hand movements: a functional magnetic resonance imaging study. Archives of physical medicine and rehabilitation. 2009 Apr 1;90(4):675-81.

37. Thirumala P, Hier DB, Patel P. Motor recovery after stroke: lessons from functional brain imaging. Neurological research. 2002 Jul 1;24(5):453-8.

38. Narayan Arya K, Pandian S. Effect of task-based mirror therapy on motor recovery of the upper extremity in chronic stroke patients: A pilot study. Topics in Stroke Rehabilitation. 2013 May 1;20(3):210-7.

39. Braun SM, Beurskens AJ, Borm PJ, Schack T, Wade DT. The effects of mental practice in stroke rehabilitation: a systematic review. Archives of physical medicine and rehabilitation. 2006 Jun 1;87(6):842-52.

40. Malouin F, Richards CL, Desrosiers J, Doyon J. Bilateral slowing of mentally simulated actions after stroke. Neuroreport. 2004 Jun 7;15(8):1349-53.

41. Slade JM, Landers DM, Martin PE. Muscular activity during real and imagined movements: a test of inflow explanations. Journal of Sport \& Exercise Psychology. 2002 Jun 1;24(2).

42. Park JH, Heo SY. A systematic review on the effect of mirror therapy on upper extremity function for stroke patients. The Journal of the Korea Contents Association. 2014;14(3):215-22.

43. Decety J, Grèzes J. Neural mechanisms subserving the perception of human actions. Trends in cognitive sciences. 1999 May 1;3(5):172-8.

44. Mehrholz J, Pohl M, Platz T, Kugler J, Elsner B. Electromechanical and robot-assisted arm training for improving activities of daily living, arm function, and arm muscle strength after stroke. Cochrane Database of Systematic Reviews. 2018(9).

45. Lioulemes A. Robot-Assisted Rehabilitation for Smart Assessment and Training. In2015 International Conference on Healthcare Informatics 2015 Oct 21 (pp. 469-469). IEEE.

46. Basteris A, Nijenhuis SM, Stienen AH, Buurke JH, Prange GB, Amirabdollahian F. Training modalities in robot-mediated upper limb rehabilitation in stroke: a framework for classification based on a systematic review. Journal of neuro engineering and rehabilitation. 2014 Dec 1;11(1):111.

47. Fitle KD, Pehlivan AU, O’Malley MK. A robotic exoskeleton for rehabilitation and assessment of the upper limb following incomplete spinal cord injury. In2015 IEEE International Conference on Robotics and Automation (ICRA) 2015 May 26 (pp. 4960-4966). IEEE. 
48. Butler DP, Willett K. Wii-habilitation: is there a role in trauma?. Injury. 2010;41(9):883-885.

49. Jack D, Boian R, Merians AS, Tremaine M, Burdea GC, Adamovich SV, Recce M, Poizner H. Virtual reality-enhanced stroke rehabilitation. IEEE transactions on neural systems and rehabilitation engineering. $2001 \mathrm{Sep} ; 9(3): 308-18$.

50. Saposnik G, Levin M, Stroke Outcome Research Canada (SORCan) Working Group. Virtual reality in stroke rehabilitation: a meta-analysis and implications for clinicians. Stroke. 2011 May;42(5):1380-6.

51. Miyamoto S, Kondo T, Suzukamo Y, Michimata A, Izumi SI. Reliability and validity of the Manual Function Test in patients with stroke. American journal of physical medicine \& rehabilitation. 2009 Mar 1;88(3):247-55.

52. Pedretti LW, Early MB, editors. Occupational therapy: Practice skills for physical dysfunction. St. Louis, MO: Mosby; 2001 Feb.

53. McCabe J, Monkiewicz M, Holcomb J, Pundik S, Daly JJ. Comparison of robotics, functional electrical stimulation, and motor learning methods for treatment of persistent upper extremity dysfunction after stroke: a randomized controlled trial. Archives of physical medicine and rehabilitation. 2015 Jun 1;96(6):98190.

54. Sanford J, Moreland J, Swanson LR, Stratford PW, Gowland C. Reliability of the Fugl-Meyer assessment for testing motor performance in patients following stroke. Physical therapy. 1993 Jul 1;73(7):447-54.

55. Woodbury ML, Velozo CA, Richards LG, Duncan PW, Studenski S, Lai SM. Longitudinal stability of the Fugl-Meyer Assessment of the upper extremity. Archives of Physical Medicine and Rehabilitation. 2008 Aug 1;89(8):1563-9.

56. Vaziri PM, Bahrpeyma F, Firoozabadi M, Forough B, Hatef B, Sheikhhoseini R, Shamili A. Low frequency repetitive transcranial magnetic stimulation to improve motor function and grip force of upper limbs of patients with hemiplegia. Iranian Red Crescent Medical Journal. 2014 Aug;16(8).
57. Granger CV, Albrecht GL, Hamilton BB. The outcome of comprehensive medical rehabilitation: measurement by PULSES profile and the Barthel Index. Archives of physical medicine and rehabilitation. 1979 Apr;60(4):145-54.

58. Jung HY, Park BK, Shin HS, Kang YK, Pyun SB, Paik NJ, Kim $\mathrm{SH}$, Kim TH, Han TR. Development of the Korean version of the Modified Barthel Index (K-MBI): Multi-center Study for Subjects with Stroke. Journal of the Korean Academy of Rehabilitation Medicine. 2007 Jun 1;31(3):283-97.

59. Carr JH, Shepherd RB. Stroke Rehabilitation. London: Butteworth Heinemann. 2003.

60. Cromwell FS. Occupational Therapist's Manual for Basic Skill Assessment. Primary Prevocational Evaluation, Fair Oaks Printing. 1976.

61. Shin HY, Lee JY, Song J, Lee S, Lee J, Lim B, Kim H, Huh S. Cause-of-death statistics in the Republic of Korea, 2014. Journal of the Korean Medical Association. 2016 Mar 1;59(3):221-32.

62. World Health Organization. Global status report on noncommunicable diseases in 2014. World Health Organization; 2014.

63. Radomski MV, Latham CA, editors. Occupational therapy for physical dysfunction. Lippincott Williams \& Wilkins; 2008.

64. Appelros P, Andersson ÅG. Changes in Mini-Mental State Examination score after stroke: lacunar infarction predicts cognitive decline. European journal of neurology. 2006 May;13(5):491-5.

65. Oros RI, Popescu CA, Iova CA, Mihancea P, Iova SO. The impact of cognitive impairment after a stroke on activities of daily living. Human and Veterinary Medicine. 2016 Mar 1;8(1):41-4.

66. Feys HM, De Weerdt WJ, Selz BE, Cox Steck GA, Spichiger R, Vereeck LE, Putman KD, Van Hoydonck GA. Effect of a therapeutic intervention for the hemiplegic upper limb in the acute phase after stroke: a single-blind, randomized, controlled multicenter trial. Stroke. 1998 Apr;29(4):785-92.

67. Youn-Bum Sung, Dae-Hwan Lee and Jung-Ho Lee. Descriptive Study on Rehabilitation Treatment Methods for Improving Upper Limb Function in Stroke Patients. International Journal of Advanced Nursing Education and Research. 2020 Aug;5(2). 